\title{
Exploring the Dependencies between Behavioral and Neuro-physiological Time-series Extracted from Conversations between Humans and Artificial Agents
}

\author{
Hmamouche Youssef ${ }^{1,2}$, Ochs Magalie ${ }^{1}$, Prévot Laurent ${ }^{2,3}$ and Chaminade Thierry ${ }^{4}$ \\ ${ }^{1}$ Aix Marseille Université, Université de Toulon, CNRS, LIS, UMR7020, Marseille, France \\ ${ }^{2}$ Aix Marseille Université, CNRS, LPL, UMR7309, Aix-en-Provence, France \\ ${ }^{3}$ Institut Universitaire de France, Paris, France \\ ${ }^{4}$ Aix Marseille Université, CNRS, INT, UMR7289, Marseille, France
}

Keywords: Multimodal Signals Processing, Conversation, Machine Learning, Human-human and Human-machine Interactions, Functional MRI.

\begin{abstract}
Whole-brain neuroimaging using functional Magnetic Resonance Imaging (fMRI) provides valuable data to localize brain activity in space and time. Here, we use a unique corpus including fMRI and behavior recorded when participants discussed with a human or a conversational robot. Temporal dynamic is crucial when studying conversation, yet identifying relationship between the participants' behavior and their brain activity is technically challenging given the time resolution of fMRI. We propose here an approach developed to extract neurophysiological and behavioral time-series from the corpus and analyse their causal relationships. Preprocessing entails the construction of discrete neurophysiological time-series from functionally well defined brain areas, as well as behavioral time-series describing higher-order behaviors extracted from synchronized raw audio, video and eyetracking recordings. The second step consists in applying machine learning models to predict brain activity on the basis of various aspects of behavior given knowledge about the functional role of the areas under scrutiny. Results demonstrate the specificity of the behaviors allowing the predictions of the activity in functional brain areas.
\end{abstract}

\section{INTRODUCTION}

In neuroimaging, signal processing and machine learning techniques have become very useful, especially in explaining or predicting the brain activity based on external signals related to behavior. Meanwhile, analysis of temporal relationships between behavior and brain activity is an important step towards the investigation of the brain bases of natural social behaviors. This requires datasets comprising synchronized neurophysiological and behavioral time series recorded during unconstrained social interactions. In this paper, we propose to develop an approach to investigate a fMRI dataset acquired when participants were having a bidirectional natural conversation with a fellow human or a conversational robot (Rauchbauer Birgit et al., 2019). This dataset is unique in that participants' behaviour is unconstrained and therefore different for each individual recording. Thus, the classical approach of contrasting two or more well controlled experimental conditions can't be used. The proposed approach consists instead in using behavioral recordings to predict fMRI responses in local-

\section{ized brain areas.}

Existing works ( $c f$. Section 2) have a major drawback in the sense that they use one or a small number of behavioral signals that are derived from very controlled tasks. Our contribution therefore consists in handling complex multimodal behavioral signals acquired during the unconstrained conversation and derive from them features that are relevant to predict brain activity in well-defined functional regions of interest. This contribution is divided in two parts. First, we propose a way of extracting representative time series(the behavioral features) from multimodal behavioral data acquired when participants discuss with a fellow human or a robot. These recordings include speech produced by the two interlocutors, as well as eyetracking signals of the participant while viewing videos of the human or artificial interlocutor. Then, we propose an approach to predict the discretized BOLD (Blood-Oxygen-Level-Dependent) signal, that measures the hemodynamic changes associated with brain activity, in well-defined functional areas from the behavioral features, using supervised machine learning algorithms. Our hypothesis is that 
only relevant behavioral features will be efficient in predicting the activity in a given brain area given domain knowledge about the function of this area.

The rest of the paper is organized as follows, after presenting existing related work (Section 2), we describe the fMRI experiment and recorded behavioral data in Section 3. Then, we formulate the problem in Section 4. We then present and discuss our results in Section 5, and summarize our approach and results in Section 6.

\section{RELATED WORK}

In the literature, many approaches based on multiple linear regression have been proposed to address the problem of fMRI signals prediction. In (Mitchell et al., 2008), the fMRI neural activation associated to meanings is predicted based on a large text data. The brain regions studied are in the sensory-motor cortex. The model used consists of transforming the meaning of text into semantic features, then building a regression model that expresses the fMRI brain activity as a linear combination of input semantic features. The authors show a prediction accuracy of 0.62 or higher, but on each participant independently. This issue has also been addressed with multi-subject approach, that is, by concatenating data from multiple subjects. For example, in (Huth et al., 2016), the goal was to predict voxels activity measured via the BOLD signal based on the speech meaning. The used data are collected from an fMRI experiment performed on 7 subjects. The brain areas predicted are from the cerebral cortex (the lateral and ventral temporal cortex, lateral and medial parietal cortex, and superior and inferior prefrontal cortex). The methodology adopted is based first on constructing predictive variables using semantic features extracted from natural language. Second, dimension reduction using Principal Component Analysis (PCA) is applied to reduce the number of the predictive variables. Then, a prediction model is learned based on multiple linear regression with regularization to predict the BOLD signal. The prediction results and the principal components of the predictive variables are both combined to classify brain areas according to the semantic features categories.

In addition to the semantic features, other behavioral signals have been analyzed by studying the effect of a single predictive variable on the brain activity. For example, the reaction time is used to predict activity in specific brain regions (Yarkoni et al., 2009). In (Chen et al., 2016), the acoustically-derived vocal arousal score ((Bone et al., 2014)) is used to predict the BOLD signal using the Gaussian mixture regression model. In (Knops et al., 2009), the authors predict the BOLD signal in the posterior parietal cortex based on eye movement data using a multivariate regression model.

More general approaches try to predict the brain activity of various areas using different types of signals at the same time. For example, in (DeSouza et al., 2012), correlations are analyzed using linear regression between the BOLD signal and behavioral features computed from observed facial expressions, reaction time and eyetracking data.

\section{DATASETS ACQUISITION AND PROCESSING}

In this section, we describe the experimental paradigm, the data recorded in this experiment, and describe our analysis. The data is collected from an fMRI experiment described in (Rauchbauer Birgit et al., 2019).

The experiment involves twenty five participants, and consists of four sessions, each containing six conversations of 60 seconds each, three with a human and three with a conversational robot alternatively. A "advertising campaign" provides a cover story: participants are informed that they should guess what is the message brought by images in which the fruits appear either as 'superheroes' or 'rotten fruits'. The conversations between the participant and either a confederate of the experimenter or a FURHAT conversational robot (Al Moubayed et al., 2012) (controlled by the confederate in a Wizard-of-Oz mode, unbeknown to the participant), are about single images of the purported "advertising campaign". The experiment design is illustrated in Figure 1 and two conversational sequences are illustrated in examples (1) (SubjectConfederate) and (2) (Subject-Furhat).

(1) C: Elle est vraiment pourrie cette framboise.

$S$ : Oui, je trouve aussi

$\mathrm{C}:$ Tu penses ce c'est pour nous faire penser à quoi?

$\mathrm{S}$ : Je sais pas, contre le gaspillage alimentaire?

C : Ouais bonne idée. [...]

(2) F: Elle est vraiment pourrie cette framboise.

$S:$ Oui, je trouve aussi

$\mathrm{F}$ : Tu penses ce c'est pour nous faire penser à quoi?

$\mathrm{S}$ : Je sais pas, contre le gaspillage alimentaire?

F : Ouais bonne idée. [...] 


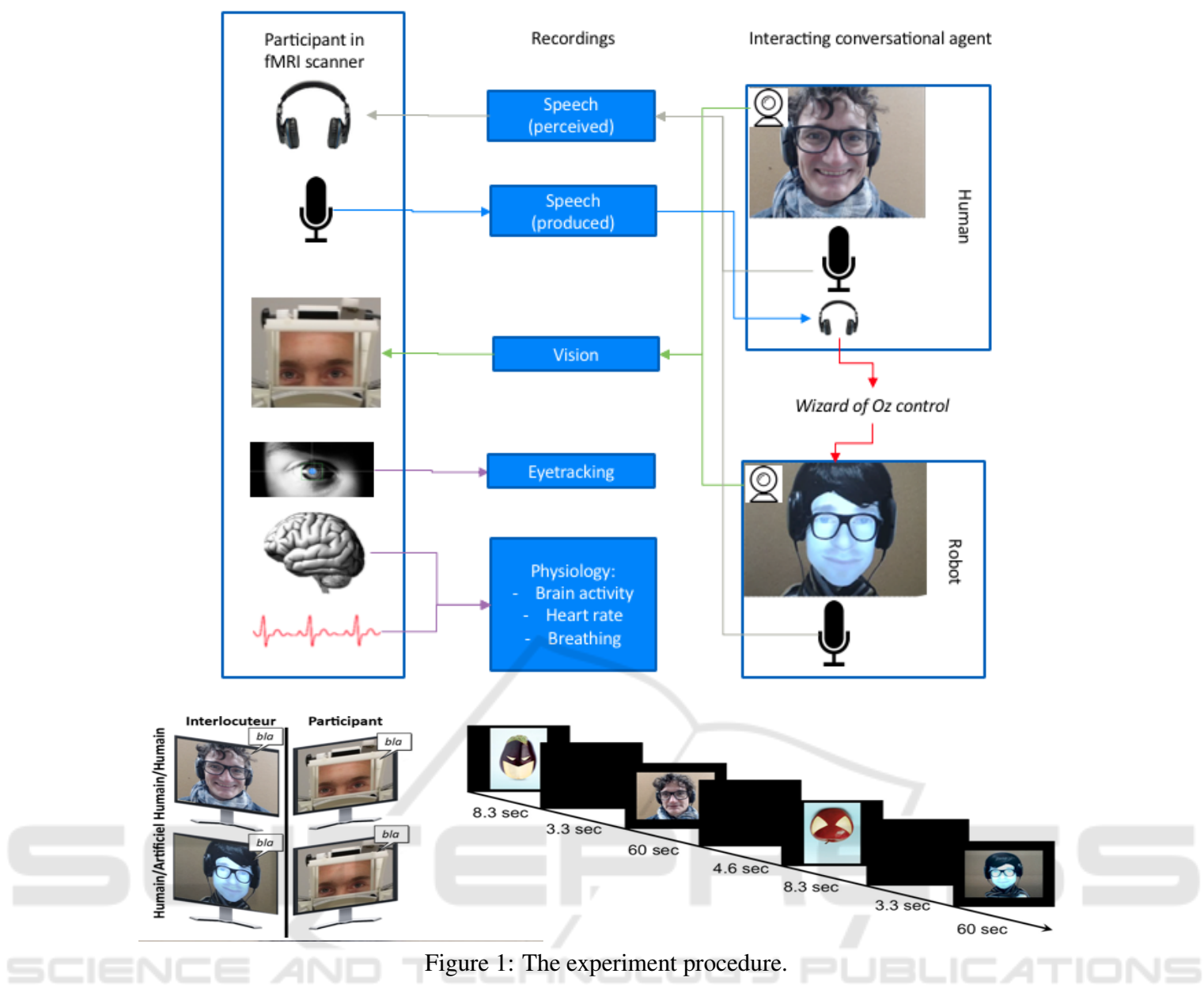

\subsection{Time Series Extraction}

We process the recorded behavioral and neurophysiological signals in order to extract representative features, then we construct structured time series for each subject that can be used for machine learning models. This processing is illustrated in Figure 3.

\subsubsection{Processing fMRI Signals}

Standard functional MRI acquisition procedures were used, described in details in (Rauchbauer Birgit et al., 2019). BOLD signal 3-dimensional images are recorded in the whole brain every 1.205 seconds. Standard SPM12 preprocessing procedures are used (Penny et al., 2011), including correction for time delays in slice acquisition ("slice timing"), image realignment, magnetic field inhomogeneities correction, normalization to the standard MNI space using the DARTEL (Ashburner, 2007) procedure for coregistration of individual participants' anatomy, and finally spatial smoothing with a 5-mm full-width halfmaximum 3-dimensional Gaussian kernel. Extraction of the BOLD signal in regions of interest is performed using the conn toolbox (Whitfield-Gabrieli and NietoCastanon, 2012), and includes several denoising procedures, firstly a linear detrending using a high-pass filter with a threshold of 128 seconds, secondly using realignment parameters to calculate nuisance regressors related to participants' movement during scanning, thirdly taking heartbeat and breathing recordings to remove physiological artifacts with the PhysIO toolbox (Kasper et al., 2017), and finally extracting BOLD signal in the white matter and cerebrospinal fluid and using the 5 first eigen variate of the timeseries as nuisance representing signal fluctuations in non-cortical brain tissues. A 275-area parcellation based on functional and anatomical connectivity patterns (Fan et al., 2016) defines regions of interest (ROI) for the whole brain, and specific regions are chosen based on their anatomical location. Continuous time-series (385 time points) are extracted for each ROI and each session and participant representing the mean activity after denoising.

For the current demonstration, we focus on 5 ROIs chosen in order to validate our approach using well- 
defined functional areas: the Fusiform Gyrus ROI corresponds to the Fusiform Face Area involved in face perception, the left and right Motor Cortex ROIs support speech production, and the left and right $\mathrm{Su}-$ perior Temporal Sulcus ROIs are involved in speech perception.

\subsubsection{Processing Multimodal Behavioral Signals}

During conversations, different types of behavioral data are recorded for both the participant and his interlocutor: video of the interlocutor, gaze movements of the participant using an eyetracking system, and the speech of both of them. For each of these modalities, several time series are computed to represent the evolution over time of different variables for both the participant and his interlocutor.

First, speech to text transformation is performed manually, then automatic annotation and segmentation are applied using SPPAS (Bigi, 2015). From the obtained transcriptions, we have extracted many linguistic time series such as the Speech activity (the presence the speech), Overlap (presence of speech of both interlocutors), Laughters, Filled-breaks and the Reaction time. The reaction time in our case represents the amount of time taken by an interlocutor to speak after the other interlocutor finishes his turn. We consider this amount positive if there is a delay between the speaking turns and negative in case of overlap.

We also consider specific features like interpersonal Particles items, i.e., words that may express the mood of the speaker (e.g., but, well, maybe), Discourses markers, which are expressions used to make the discourse organized (e.g., I mean, so, therefore, okay) (Schiffrin, 1987), and Feedback lexical items, which represent words used for reaction, perception and understanding (e.g., yes, no, okay, right) (Gravano et al., 2011).

The time series categorizing these features are resampled according to the fMRI acquisition frequency by considering the percentage of their existence in each time bin. Figure 2 illustrates an example of resampling the speech activity. Note that if we make direct projection on the expected axis, we may lose information between two consecutive fMRI acquisitions concerning the quantity of speech or silence that have occurred. That is why at each point we consider the percentage to have a sort of summary of what happens after the previous point.

The speeches are also analyzed via lexical richness based on two metrics from (Ochs et al., 2018), that consider the number of the different words (typetoken ratio) and the number of adjectives plus the number of adverbs resp., divided by the number of to-

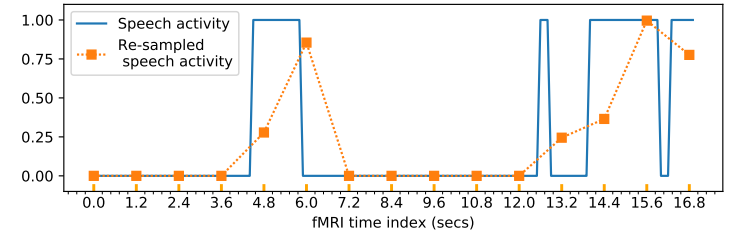

Figure 2: Example of resampling speech features (the speech activity).

tal words in the text of each speaking turn. Sentiment analysis is also considered by calculating the polarity and the subjectivity using the Pattern library (Smedt and Daelemans, 2012). The polarity score fluctuates between -1 (negative behavior) and 1 (positive behavior), while the subjectivity is between 0 (objective) and 1 (personal). The method of their calculations is based first on manual association of the polarity and the subjectivity scores to a set of adjectives among the most used. Second, another set is extracted with the most frequent nouns and the predecessor adjectives as features. Finally, a kNN classifier is learned to determine the scores of neighbor adjectives of those manually annotated (Smedt and Daelemans, 2012).

From videos, we used pre-trained models from Openface (Baltrusaitis et al., 2018) to extract 68 facial landmarks and 17 facial action units, which categorize facial movements (Bartlett et al., 1996). The $3 D$ coordinates of gaze movements ( 3 features) and head pose translations and rotations (6 features) are also extracted. The time series associated with these features are constructed by analyzing each image of the videos. In this case, the resampling task is less difficult compared to speech features, because all the variables have the same frequency and the same number of observations. From raw eyetracking data, we compute the speed of the gaze movements. Then, we project the gaze coordinates on visual stimulation using the extracted landmarks to localize where the subject is looking in at each time step (face, eyes, mouth). The saccades are also detected by the used eyetracking system, and added to the extracted features.

Table 1 summarizes all the extracted predictive features. After gathering and resampling them in addition to the BOLD signal, we build multivariate time series for each subject with the same number of observations.

\section{ANALYTICAL APPROACH}

One hypothesis of this work is that the activity in functional brain areas is mainly determined by what participant experiences, described here by behavioral signals. Therefore, we try to predict the processed 
Table 1: The extracted behavioral features.

\begin{tabular}{l|l}
\hline Modality & Features \\
\hline Speech & $\begin{array}{l}\text { Speech activity, Particles items, } \\
\text { Discourse Markers, Overlap, Re- } \\
\text { action Time, Filled-breaks, Feed- } \\
\text { backs, Laughters, Lexical Rich- } \\
\text { ness, Polarity, Subjectivity. } \\
\text { Facial Action Units (17 vari- } \\
\text { ables), Head pose coordinates (6 } \\
\text { variables), Gaze coordinates (3 } \\
\text { variables). } \\
\text { Gaze movement of the partici- } \\
\text { pant (2 variables), and 4 binary } \\
\text { variables categorizing resp. the } \\
\text { presence of saccades, and if the } \\
\text { participant is looking at the face, } \\
\text { the eyes of the mouth of the inter- } \\
\text { locutor. }\end{array}$ \\
\hline
\end{tabular}

\section{Raw Signals}

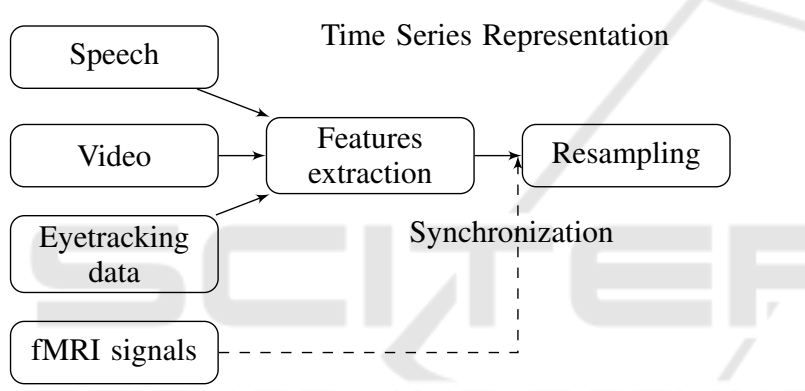

Figure 3: Extracting structured time series from raw multimodal signals.

BOLD signal based only on the history of behavioral features. Note that it is possible to predict the activity of a brain area based on its previous observations and the previous observations of other related areas. Accordingly, auto-regressive models can be used in this situation, such as the ARIMA (Auto-Regressive Integrated Moving Average) (Box et al., 2015) model to predict a single time series, or the co-intregrated vector auto-regressive models for multivariate prediction of non-stationary time series (Johansen, 1991). In our research work, our goal is to better understand the behavioral features related to the brain activity by comparing human-human and human-machine interaction. Consequently, we focus on the behavioral features to predict the BOLD signal.

\subsection{Feature Selection}

Domain knowledge allows us to determine the set of possible behavioral signals responsible for the activation of each brain area. For example, to predict the BOLD signal of the Fusiform Gyrus area, which is involved in face perception (Kanwisher and Yovel, 2006), we can use features derived from video and eyetracking signals, in order to take into account the visual simulation and the gaze movement of the participant to evaluate where he is looking. For some complex brain areas, this is not sufficient, especially when multiple modalities are involved, because the set of generated predictive features may be large. In this case, we use automatic feature selection to refine the input features before applying prediction models.

\subsection{Prediction}

The BOLD signal results from a function called the Hemodynamic Response Function (HRF), which determines the activation delay after receiving a trigger event, which peaks close to 5 seconds after the event (Gössl et al., 2001). This delay is a key parameter in our approach to model the dynamic between the BOLD signal and the behavioral features. Let $Y(t)$ be a variable representing the discretized BOLD signal of a given brain area of one subject, and $X(t)=\left\{X_{1}(t), X_{2}(t), \ldots, X_{k}(t)\right\}$, is a $k$-dimensional time series representing the behavioral variables. The first formulation that comes to mind is to expresses each value of the BOLD signal at time $t$ as a function of the predictive features at time $t-5 s$. This process can be written as follows:

$Y(t)=f\left(X_{1}(t-5 s), X_{2}(t-5 s), \ldots, X_{k}(t-5 s)\right)+U(t)$,

where $U(t)$ is the vector of errors of the model, and $f$ is the function that we want to find.

Considering the fact that the $5 s$ delay is not fix for all subjects and brain areas, but varies around $5 s$, it can be more relevant to include more than one values around the $5 s$. Consequently, as an improvement of equation 1 , our formulation can be expressed as follows:

$$
\begin{aligned}
Y(t)= & f\left(X_{1}(t-4 s), X_{1}(t-5 s), X_{1}(t-6 s),\right. \\
& \ldots, \\
& X_{k}(t-4 s), X_{k}(t-5 s), X_{k}(t-6 s)+U(t),
\end{aligned}
$$

As mentioned in Section 1, our approach is based on discretizing the variable describing local brain activity into binary variable (activation or not). The motivation behind the discretization is that instead of predicting the exact value of the BOLD signal, we start by predicting if a brain area is active or not. In our case, classification models can be used to approximate the function $f$. The other possibility is to keep the BOLD signals continuous and use multiple regression to predict them, which is the classical approach 
in the literature (Mitchell et al., 2008; Huth et al., 2016; Knops et al., 2009; DeSouza et al., 2012). In our case, we evaluate both approaches by using multiple regression, then discretizing the predictions in order to compare all models with same prediction accuracy measures.

\section{RESULTS}

In this section, we present the prediction procedure used, the obtained prediction scores, and discuss the results.

\subsection{Prediction Procedure}

The pre-processed time series of each subject are restructured in such a way to have a target variable and the lagged behavioral variables as predictive features according to Equation 2. The data of all subjects are concatenated, and randomly shuffled to train and test the models on all observations. The prediction procedure is based on a 10-fold-cross validation to find the parameters of the prediction models on the training set ( $80 \%$ of the data). Second, feature selection and dimension reduction methods are applied with different reduction sizes to find the most relevant lagged variables. Her we evaluate two methods, the Recursive Feature Elimination (Guyon et al., 2002) and the Principal Component Analysis (Tipping and Bishop, 1999). Afterwards, prediction models are tested on $20 \%$ of the data. This procedure is repeated 5 times to test the models on all data. Finally, we select the model and the predictive features leading to the best prediction scores for each brain area.

The experiments are conducted on human-human and human-robot conversations separately in order to compare the differences between the two conditions in terms of the selected behavioral features activating specific brain areas. In both cases, the data (training and test sets) consists of 9180 observations. In this paper, we concentrate on five brain areas: Fusiform Gyrus Area involved in face perception, left and right Motor Cortex for speech production, and left and right Superior Temporal Sulcus for speech perception and social cognition. These areas are chosen in order to validate our approach before investigating all brain areas in future works.

We evaluated classical classifiers using the Sickitlearn machine learning library (Pedregosa et al., 2011): SVM, Random Forest (RF), and Gradient Boosting (GB). The RIDGE model (regression with regularization) is also evaluated with the idea of predicting the continuous BOLD signal, then discretiz-

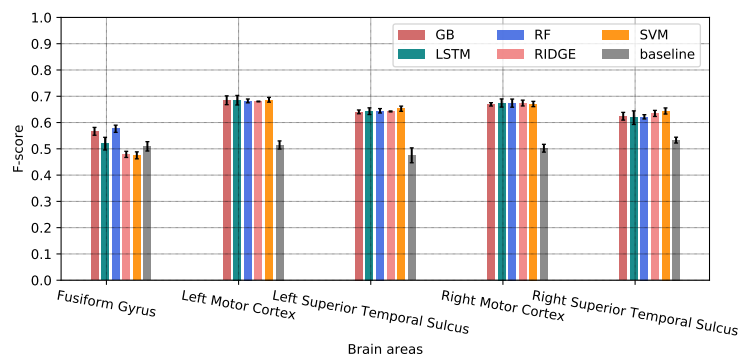

(a)

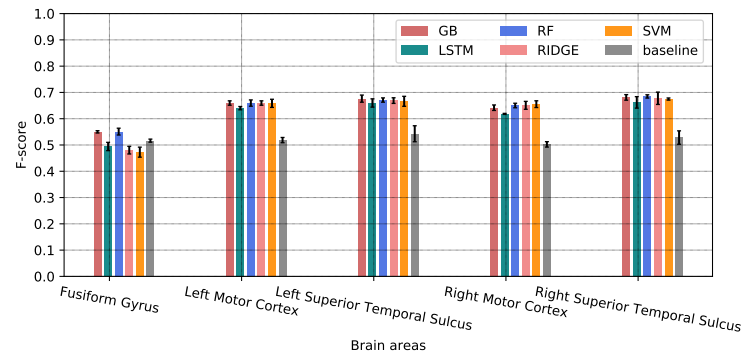

(b)

Figure 4: Prediction accuracy for human-human (a) and human-robot (b) interactions, based of the F-score measures. The values represent the mean of the F-scores over 5 prediction experiments, while the error bars represent the standard deviations.

ing the predictions. We use also the Long Short Term Memory (LSTM) network from the Tensorflow library (Abadi et al., 2016). Finally, a baseline classifier is evaluated with random predictions using 3 strategies: stratified, most frequent, and uniform, where the appropriate strategy of each target variable is chosen in the training step.

\subsection{Prediction Scores}

Figure 4 shows a comparison between the evaluated classifiers in terms of the mean and the standard deviation of the weighted F-score over the 5 prediction tests. 1 The results show that globally almost all models outperform the baseline, and they are close for brain areas involved in speech perception and production. Overall, the best f-scores are between 0.65 and 0.7, except for the Fusiform Gyrus area that we found the most difficult to predict, where only the Random Forest Gradient Boosting classifier that are slightly better than the baseline.

\footnotetext{
${ }^{1}$ We only showed the results based on f-score as it resumes the precision and the recall. These measures are also calculated, more details about their results in addition to the scores of each subset of behavioral features can be found in https://github.com/Hmamouche/NeuroTSConvers.
} 


\subsection{Discussion}

The proposed prediction process allows to identify the features that elicit the local activation of the brain areas, and to compare the difference between the situations where a participant is talking with a human or a robot. For the Fusiform Gyrus area (face perception), the selected features for human-human conversations are head movements and facial action units with the random forest classifier, while from humanmachine conversations, eyetracking features (i.e., the speed of gaze coordinates, saccades and whether the participant is looking at the face of the robot) are also needed. This can be explained by the fact that our scenario for the talking head does not express significant facial movements compared to the human. For left and right Motor cortex areas, which are involved in speech production, the obtained results confirm the hypothesis that the best predictions are obtained using only the speech activity of the participant. For these areas, we found no difference between human-human and human-machine conversations. For left and right Motor cortex areas, the obtained results confirm the hypothesis that the best predictions are obtained using only the speech activity of the participant. For these areas, we found no difference between human-human and human-machine conversations.

For left and right Superior Temporal Sulcus, only the Speech activity of the interlocutor is needed for human-robot interactions. For human-human interactions, different linguistics features are selected for the right $\{$ Speech activity, Filled-breaks, Feedbacks, and Discourses markers\}, compared to the left area, for which just the Speech activity is required. These brain areas are an interesting example to compare humanhuman and human-machine, as we see the absence of social cognition features where the interlocutor is a robot.

Let us note that each prediction model selects its own best predictors for each brain area. Nevertheless, there is some sort of stability in this selection over all models. For example, all the models select the speech activity of the participant to predict left and right Motor Cortex areas. They differs a little for left and right Superior Temporal Sulcus. For instance, the Random Forest model selects linguistics features involved in perception, while the SVM includes some facial action units. One explanation might be that different behavioral features may provide the same predictive information. In our case, we select the final best predictors based on the best model for each brain area.

We can conclude that the local activation of the studied brain areas involved in speech production and perception can be predicted, allowing us to identify causal dependencies between behavioral and neuro- physiological time series, and compare human-human and human-machine interactions. Importantly, the relevant features are in complete agreement with the social cognitive neuroscience literature. One limit that we face concerns the prediction of the Fusiform Gyrus area activity. Although the features selected to predict the activity of this area are logical, improving the prediction performances requires further investigation, and particularly to compare prediction for the human and robot agent given the differences in their head movements and facial expression.

\section{CONCLUSION}

In this paper, we propose an approach to predict the activity in specific brain areas based on the multi-modal behavioral signals of human-human and human-machine conversations. This is a worthwhile alternative to the classical approach that consists in predicting continuous fMRI signals directly using multiple regression. The results show that the discretized BOLD signals of brain areas involved in speech perception and production are predictable based only on linguistics time series. In future works, we plan to explore all brain regions and to define higher-order behavioral features. The approach proposed here confirms the links between behavioral variables and the functional brain areas under scrutiny, but more importantly paves the way to discover new dependencies between behaviour and local activity across the whole brain in a natural social interaction.

\section{REFERENCES}

Abadi, M., Barham, P., Chen, J., Chen, Z., Davis, A., Dean, J., Devin, M., Ghemawat, S., Irving, G., Isard, M., et al. (2016). Tensorflow: A system for largescale machine learning. In 12th $\{$ USENIX $\}$ Symposium on Operating Systems Design and Implementation ( $\{O S D I\} 16)$, pages 265-283.

Al Moubayed, S., Beskow, J., Skantze, G., and Granström, B. (2012). Furhat: A back-projected human-like robot head for multiparty human-machine interaction. In Esposito, A. e. a., editor, Cognitive Behavioural Systems, Lecture Notes in Computer Science, pages 114 130. Springer Berlin Heidelberg.

Ashburner, J. (2007). A fast diffeomorphic image registration algorithm. NeuroImage, 38(1):95-113.

Baltrusaitis, T., Zadeh, A., Lim, Y. C., and Morency, L. (2018). OpenFace 2.0: Facial Behavior Analysis Toolkit. In 2018 13th IEEE International Conference on Automatic Face Gesture Recognition (FG 2018), pages 59-66. 
Bartlett, M. S., Viola, P. A., Sejnowski, T. J., Golomb, B. A., Larsen, J., Hager, J. C., and Ekman, P. (1996). Classifying facial action. In Advances in neural information processing systems, pages 823-829.

Bigi, B. (2015). SPPAS - multi-lingual approaches to the automatic annotation of speech. The Phonetician, 111-112(ISSN:0741-6164):54-69.

Bone, D., Lee, C.-C., and Narayanan, S. (2014). Robust Unsupervised Arousal Rating: A Rule-Based Framework with Knowledge-Inspired Vocal Features. IEEE transactions on affective computing, 5(2):201-213.

Box, G. E., Jenkins, G. M., Reinsel, G. C., and Ljung, G. M. (2015). Time series analysis: forecasting and control. John Wiley \& Sons.

Chen, H., Liao, Y., Jan, H., Kuo, L., and Lee, C. (2016). A Gaussian mixture regression approach toward modeling the affective dynamics between acousticallyderived vocal arousal score (VC-AS) and internal brain fMRI bold signal response. In 2016 IEEE International Conference on Acoustics, Speech and Signal Processing (ICASSP), pages 5775-5779.

DeSouza, J. F., Ovaysikia, S., and Pynn, L. K. (2012). Correlating Behavioral Responses to fMRI Signals from Human Prefrontal Cortex: Examining Cognitive Processes Using Task Analysis. Journal of Visualized Experiments : JoVE, (64).

Fan, L., Li, H., Zhuo, J., Zhang, Y., Wang, J., Chen, L., Yang, Z., Chu, C., Xie, S., Laird, A. R., Fox, P. T., Eickhoff, S. B., Yu, C., and Jiang, T. (2016). The Human Brainnetome Atlas: A New Brain Atlas Based on Connectional Architecture. Cereb Cortex, 26(8):3508-3526.

Gössl, C., Fahrmeir, L., and Auer, D. (2001). Bayesian modeling of the hemodynamic response function in bold fmri. NeuroImage, 14(1):140-148.

Gravano, A., Hirschberg, J., and Beňuš, Š. (2011). Affirmative cue words in task-oriented dialogue. Сотриtational Linguistics, 38(1):1-39.

Guyon, I., Weston, J., Barnhill, S., and Vapnik, V. (2002). Gene selection for cancer classification using support vector machines. Machine learning, 46(1-3):389-422.

Huth, A. G., de Heer, W. A., Griffiths, T. L., Theunissen, F. E., and Gallant, J. L. (2016). Natural speech reveals the semantic maps that tile human cerebral cortex. $\mathrm{Na}$ ture, 532(7600):453-458.

Johansen, S. (1991). Estimation and hypothesis testing of cointegration vectors in gaussian vector autoregressive models. Econometrica: journal of the Econometric Society, pages 1551-1580.

Kanwisher, N. and Yovel, G. (2006). The fusiform face area: a cortical region specialized for the perception of faces. Philosophical Transactions of the Royal Society B: Biological Sciences, 361(1476):2109-2128.

Kasper, L., Bollmann, S., Diaconescu, A. O., Hutton, C., Heinzle, J., Iglesias, S., Hauser, T. U., Sebold, M., Manjaly, Z.-M., Pruessmann, K. P., and Stephan, K. E. (2017). The PhysIO Toolbox for Modeling Physiological Noise in fMRI Data. Journal of Neuroscience Methods, 276:56-72.
Knops, A., Thirion, B., Hubbard, E. M., Michel, V., and Dehaene, S. (2009). Recruitment of an Area Involved in Eye Movements During Mental Arithmetic. Science, 324(5934):1583-1585.

Mitchell, T. M., Shinkareva, S. V., Carlson, A., Chang, K.-M., Malave, V. L., Mason, R. A., and Just, M. A. (2008). Predicting Human Brain Activity Associated with the Meanings of Nouns. Science, 320(5880):1191-1195.

Ochs, M., Jain, S., and Blache, P. (2018). Toward an automatic prediction of the sense of presence in virtual reality environment. In Proceedings of the 6th International Conference on Human-Agent Interaction, pages 161-166. ACM.

Pedregosa, F., Varoquaux, G., Gramfort, A., Michel, V., Thirion, B., Grisel, O., Blondel, M., Prettenhofer, P., Weiss, R., Dubourg, V., et al. (2011). Scikitlearn: Machine learning in python. Journal of machine learning research, 12(Oct):2825-2830.

Penny, W. D., Friston, K. J., Ashburner, J. T., Kiebel, S. J., and Nichols, T. E. (2011). Statistical Parametric Mapping: The Analysis of Functional Brain Images. Elsevier.

Rauchbauer Birgit, Nazarian Bruno, Bourhis Morgane, Ochs Magalie, Prévot Laurent, and Chaminade Thierry (2019). Brain activity during reciprocal social interaction investigated using conversational robots as control condition. Philosophical Transactions of the Royal Society B: Biological Sciences, 374(1771):20180033.

Schiffrin, D. (1987). Discourse markers. Number 5. Cambridge University Press.

Smedt, T. D. and Daelemans, W. (2012). Pattern for python. Journal of Machine Learning Research, 13(Jun):2063-2067.

Tipping, M. E. and Bishop, C. M. (1999). Probabilistic principal component analysis. Journal of the Royal Statistical Society: Series B (Statistical Methodology), 61(3):611-622.

Whitfield-Gabrieli, S. and Nieto-Castanon, A. (2012). Conn: A Functional Connectivity Toolbox for Correlated and Anticorrelated Brain Networks. Brain Connectivity, 2(3):125-141.

Yarkoni, T., Barch, D. M., Gray, J. R., Conturo, T. E., and Braver, T. S. (2009). BOLD Correlates of Trial-byTrial Reaction Time Variability in Gray and White Matter: A Multi-Study fMRI Analysis. PLOS ONE, 4(1):e4257. 